\title{
Kernos
}

Revue internationale et pluridisciplinaire de religion grecque antique

5 | 1992

Varia

\section{Le mot et les rites. Aperçu des significations de ópyı et de quelques dérivés}

\section{André Motte et Vinciane Pirenne-Delforge}

\section{CpenEdition \\ Journals}

\section{Édition électronique}

URL : http://journals.openedition.org/kernos/1055

DOI : 10.4000/kernos. 1055

ISSN : 2034-7871

\section{Éditeur}

Centre international d'étude de la religion grecque antique

\section{Édition imprimée}

Date de publication : 1 janvier 1992

ISSN : 0776-3824

\section{Référence électronique}

André Motte et Vinciane Pirenne-Delforge, « Le mot et les rites. Aperçu des significations de ópyıa et de quelques dérivés », Kernos [En ligne], 5 | 1992, mis en ligne le 19 avril 2011, consulté le 02 mai 2019. URL : http://journals.openedition.org/kernos/1055; DOI : 10.4000/kernos.1055 


\section{LE MOT ET LES RITES. APERCU DES SIGNIFICATIONS DE "OPTIA ET DE QUELQUES DÉRIVÉS}

S'il s'est trouvé des hellénistes pour participer à un colloque sur ce thème, c'est assurément qu'ils avaient tous une notion plus ou moins claire, ou plus ou moins confuse, de ce que l'on appelle l'«élément orgiastique». Mais la signification de cette expression, directement issue d'un mot grec, correspond-elle à une notion qu'avaient les Anciens eux-mêmes, coïncide-t-elle, en particulier, avec ce qu'ils

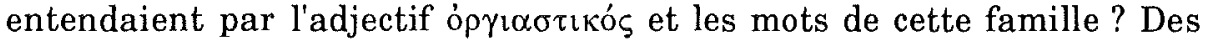
enquêtes de ce type, concernant d'autres notions, ont vu le jour depuis quelques années. Elles sont loin d'être vaines et aboutissent à des résultats intéressants et parfois surprenants. Un exemple très récent puisqu'il s'agit d'un article de Claude Calame publié dans le numéro 4 de cette même revue - montre, après d'autres, combien notre notion de mythe, qui puise son origine formelle dans la langue grecque, est loin de correspondre au champ sémantique de $\mu \hat{v} \theta 0 \varsigma^{1}$.

On ne répétera jamais assez combien il est hasardeux et méthodologiquement contestable de projeter sans autre précaution nos habitudes mentales sur les conceptions religieuses des Anciens, surtout lorsque les outils conceptuels et les catégories interprétatives dont on use plongent leurs racines dans la langue du peuple étudié. Il n'est pas inutile, en l'occurrence, de vérifier la pertinence de nos instruments de travail, quitte à jeter a priori, ne serait-ce qu'un bref moment, un "soupçon" sur la pertinence même de notre rassemblement arcadien...

Ainsi conviendra-t-on de se méfier des traductions françaises qui utilisent les mots "orgie", "orgiaque»; les connotations d'excès et de démesure qui leur sont immanquablement liées ne s'adaptent guère à la signification la plus courante de leurs correspondants grecs. De même, et à l'inverse, il n'est pas rare de trouver sous la plume des interprètes des considérations du type «tel emploi d'orgia apparaît dans un contexte "non orgiastique». Or que peut bien signifier pareille affirmation si elle ne s'accompagne d'une précision sur ce que l'on entend par "orgiastique»?

1 C. CALAME, Mythe et rite, in Kernos, 4 (1991), p.179-204. 


\section{Un regard sur les Modernes}

La grande majorité des tentatives de définition du terme óp s'appuie sur l'étymologie : tirant ő $\gamma \iota \alpha$ de la même racine que ě $\rho \omega$,

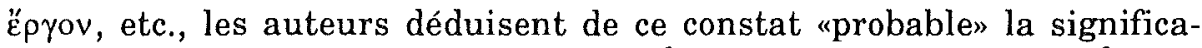
tion originelle de «ce qui est accompli ${ }^{2}$, acte cultuel en général ${ }^{3}$, rite religieux ${ }^{4}$. Le terme se serait spécialisé dans la suite pour désigner de préférence les cérémonies de certains cultes à mystère, et tout particulièrement les mystères dionysiaques. Nous aurons l'occasion de nous interroger sur la pertinence de telles assertions.

Il n'existe à ce jour qu'une seule tentative d'analyse systématique de la famille de óp $\gamma$ i $\alpha$. On la doit à Van den Burgh, un savant hollandais qui, dans une thèse publiée en 1939, a examiné les termes ó $\pi$ a $\rho \eta \eta \tau \alpha$,

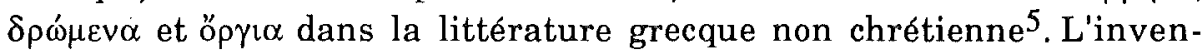
taire qu'il propose des passages concernés n'est pas sans intérêt mais, outre que l'informatique permet aujourd'hui de tendre vers une plus grande exhaustivité, on peut déplorer l'absence de toute analyse un peu approfondie. Les citations d'auteurs se juxtaposent dans un ordre chronologique qui distingue l'“avant» et l'«après» Alexandre pour chaque famille de mots étudiée. Non seulement, chacune d'elles ne reçoit pas de traitement adéquat comme telle, mais de plus, aucun essai de mise en perspective des trois familles de mots n'est même amorcé. Enfin, les attestations épigraphiques ne sont pas prises en considération dans l'inventaire.

Il n'était donc pas inutile de remettre l'ouvrage sur le métier.

2 L. BRuit-Zaidman, P. Schmitt-PANTEL, La religion grecque dans la cité grecque à l'époque classique, Paris, 1991² [1990], p. 169.

3 L. Gernet, Le génie grec dans la religion, Paris, $1970^{2}$ [1932], p. 125; J. Labarbe, in P. Poupard (éd.), Dictionnaire des religions, Paris, 1985, p. 12391240.

4 L. Ziehen, art. Orgia, in RE, XVIII, 1 (1939), c. 1026-1029; P. ChanTRAINe, Dict. étym. langue grecque, III, Paris, 1974, p. 816, s.v. ö $\rho \gamma$ ı. Cf. M. MEIERBRUgGer, $Z u$ griechisch ö $p \gamma \mathrm{l} \alpha$, in Historische Sprachforschung, 101 (1988), p. 104-107.

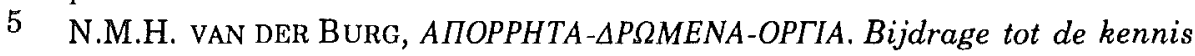
der religieuze terminologie in het Grieksch, Amsterdam, 1939. 


\section{Le corpus littéraire rassemblé}

L'interrogation du Thesaurus Linguae Graecae ${ }^{6}$ à l'aide du logiciel Pandora nous a fourni quelque trois cent cinquante occurrences d'öp pro et des mots apparentés, soit plus du double des occurrences prises en compte par Van den Burgh. Dressons-en rapidement l'inventaire.

Substantifs :

öpri $\alpha$ est de beaucoup le mieux représenté : 207 attestations, dont la plus ancienne figure dans l'Hymne hom. à Déméter; à noter que öpriov apparaît quatre fois au singulier;

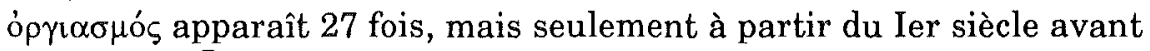
notre ère ${ }^{7}$;

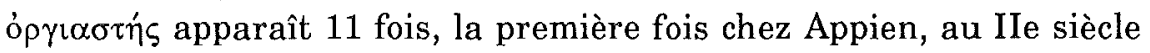
de notre ère;

óproøóvins apparaît 4 fois, dans deux épigrammes de l'Anthologie palatine et deux hymnes orphiques;

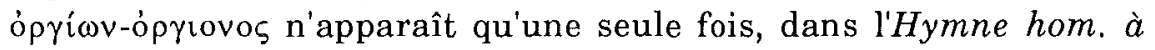
Apollon.

Verbes :

ópyı́ó $\zeta \omega$ apparait 65 fois; les premières attestations se trouvent chez Euripide, Platon et Isocrate;

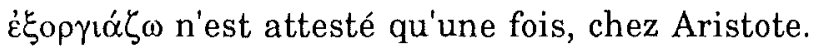

$\kappa \alpha \tau \circ \rho \gamma \_\alpha ́ \zeta \omega$ est essentiellement employé par Plutarque qui rassemble 5 attestations sur 6 ;

$\sigma u v o p \gamma\llcorner\alpha \dot{\zeta} \omega$ est lui aussi tardif et peu représenté ( 3 fois : Plutarque,

Aristide Quintilien et Synésios de Cyrène).

Adjectifs :

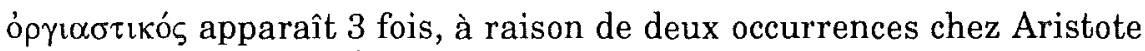
et une chez Strabon, cette dernière fournissant la forme au nominatif neutre singulier précédée de l'article;

6 La banque de données TLG comprend les deux tiers de toute la littérature en langue grecque, en ce compris les auteurs chrétiens écrivant en grec. Cf. L. Berkowitz, K.A. Squitier, Thesaurus Linguae Graecae. Canon of Greek Authors and Works, Oxford, $1986^{2}$ [1977].

7 Le fragment 985 des Poetae Melici Graeci de D.L. PAGE (Fragmenta Adespota,

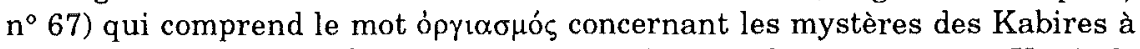
Lemnos était jadis attribué à Pindare, ce qui aurait fait remonter au Ve siècle av. J.-C. la première attestation du mot. 


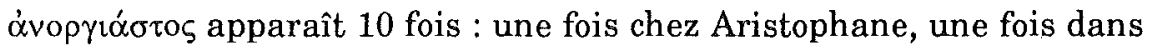

l'Épinomis de Platon, et 8 fois chez des auteurs plus tardifs;

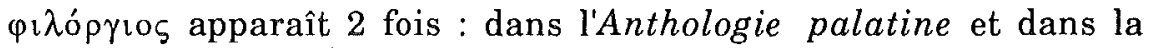

paraphrase de l'Évangile de St Jean par Nonnos;

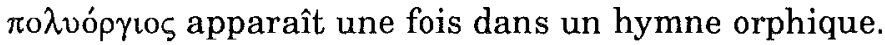

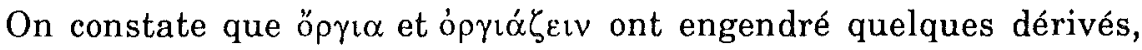

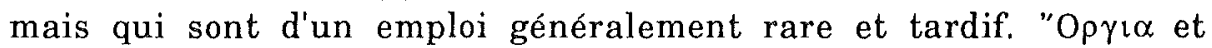

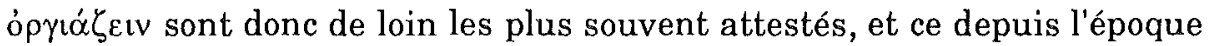
archaïque.

Il faut aussi remarquer d'emblée que cette famille de mots n'apparaît ni chez Homère, ni chez Hésiode. Deux explications sont possibles; la première, peu vraisemblable, voudrait que le mot n'existât pas encore à l'époque; la seconde, plus probable, suggère que cette absence tient à la nature même des réalités religieuses désignées par öp $\gamma \iota \alpha$ et ses parents; Dionysos et Déméter, qui sont les divinités de beaucoup les plus souvent concernées par les attestations, sont quasiment absents de l'épopée homérique, tandis que la Théogonie hésiodique se prête peu à des développements sur le culte des divinités dont est décrite la venue au jour ${ }^{8}$; il n'est dès lors pas autrement étonnant que les óp $ү \alpha$ n'aient pas trouvé place dans les plus anciens textes grecs.

Ce constat de carence à date ancienne laisse également rêveur quant à l'affirmation d'un prétendu «sens général» qu'aurait originellement

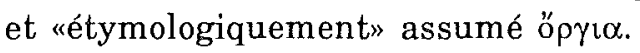

\section{Objectifs de l'étude et méthode mise en œuvre}

Le but de cette communication est de mettre en lumière la ou les signification(s) d'ópyı et, si possible, de ses dérivés. Pour ce faire, il serait nécessaire d'exploiter systématiquement tout le corpus rassemblé en posant aux textes la même question qu'adresse Penthée à Dionysos dans les Bacchantes d'Euripide ${ }^{9}$, mais que le dieu laisse sans réponse : «les öp $\gamma \iota \alpha$, dis-moi, quelle en est pour toi la nature ?».

En première approximation, il apparaît que le pluriel ő $p \gamma \iota$ est utilisé au moins dans deux sens. Le plus souvent, il signifie un ensemble d'actes rituels dont il faudra essayer de déterminer s'il s'agit d'un complexe d'un type particulier. Dans un certain nombre de cas, il

8 Les Travaux et les Jours proposent une approche différente des réalités religieuses, mais le mot n'y apparaît pas non plus.

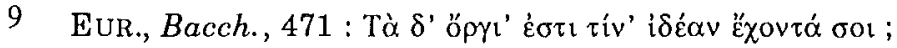


désigne manifestement des objets concrets servant à des célébrations. Ces derniers emplois ne sont pas rares, et l'on remarquera que c'est notamment le cas des quelques attestations, tardives il est vrai, d'óp au singulier. Mais distinguer entre ces deux significations n'est pas toujours aisé, d'autant que la compréhension du terme comme «célébration» a nettement primé parmi les interprètes modernes. Pour affiner la définition, on a été attentif aux relations qu'entretiennent óp $\gamma \iota \alpha$ et ses dérivés avec d'autres mots. L'examen du champ sémantique doit permettre le cas échéant de répondre à une série de questions :

1. quels sont les dieux particulièrement concernés ?

2. quels actes ou quels objets particuliers le mot őpyı désigne-t-il ?

3. quels desservants et quels fidèles prennent part aux célébrations et de quelle nature est leur participation?

4. y a-t-il des lieux de prédilection pour ces cultes et des espaces symboliques sont-ils particulièrement adaptés à l'image des óp $\gamma\llcorner\alpha$ ?

5. les öp $\gamma \iota \alpha$ supposent-ils un temps privilégié ?

6. et, en manière de synthèse, est-il un type particulier de pensée religieuse dont relèvent les manifestations cultuelles en question?

Ces questions ne sont pas exhaustives et il est évident que tous les textes n'apportent pas une réponse à chacune d'entre elles. Parfois, c'est la prise en compte d'un contexte plus large qui permet d'apporter des éclaircissements. Il va sans dire que l'espace d'une communication ne peut contenir l'analyse minutieuse d'un aussi grand nombre d'attestations. Aussi avons-nous choisi de mettre l'accent sur les époques archaïque et classique, en y ajoutant le IIIe siècle av. J.-C. (une cinquantaine d'emplois), et de confronter ponctuellement les résultats obtenus avec certains textes postérieurs, qu'il s'agisse de tentatives de théorisation déjà présentes dès l'Antiquité, de passages où les réalités religieuses sont particulièrement décantées et donc bien mises en lumière, ou de dénonciations apologétiques par des auteurs chrétiens. Il nous est apparu, enfin, que le critère le plus englobant était celui des divinités concernées, et c'est donc en fonction de ce critère que nous avons choisi de présenter les résultats de notre enquête. Dans un second temps seront examinés les emplois sans relation apparente avec un dieu précis. 


\section{Déméter : les Mystères d'Éleusis et les Thesmophories}

La première place revient à Déméter; d'une part, c'est elle qui est concernée par les premières attestations d'öp $\gamma ı \alpha$, au cœur du vieil hymne homérique qui lui est consacré, d'autre part, cette association demeure privilégiée dans la suite. Les deux premiers emplois d'őp $\rho\llcorner\alpha$ sont directement liés à la fondation des mystères d'Éleusis. La déesse, qui a ordonné aux habitants de la cité l'établissement d'un temple, annonce qu'elle "placera", qu'elle "enseignera» - la forme verbale utilisée est vं $\pi 0 \theta \eta ் \sigma o \mu \alpha$ - les ö $\gamma_{1} \alpha$ afin que les fidèles lui rendent grâce en «agissant

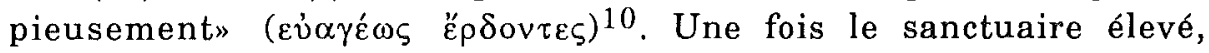
Déméter en deuil s'y réfugie, mais il faut attendre le retour de Korè du royaume d'Hadès pour que la déesse accomplisse sa promesse en divul-

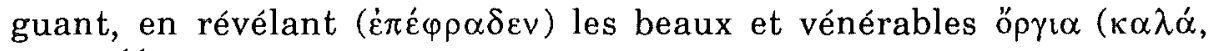

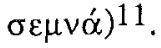

Pour la compréhension de ces deux emplois, il convient de ne pas négliger l'éventualité du caractère matériel des óp yı offerts aux hommes par la déesse. Dans le premier cas, l'ordre de construire un

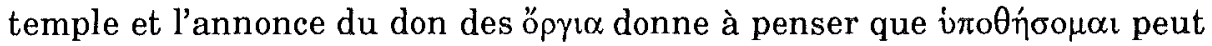
être compris dans le sens de "placer", le bâtiment devenant une sorte d'écrin pour les objets sacrés de la déesse. Dans cette hypothèse, comment ne pas évoquer l'anaktoron qui abritait les objets sacrés au sein du télestérion? Dans le second cas, on a une double expression : la déesse enseigne $(\delta \varepsilon \hat{\imath} \hat{\xi} \varepsilon)$ le déroulement des rites ( $\left.\delta \rho \eta \sigma \mu \circ \sigma u ́ v \eta v \theta^{\prime}{ }^{\prime} \varepsilon \rho \hat{\omega} v\right)$ et

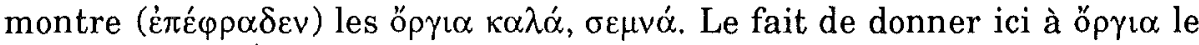
sens concret d'“objet" présente l'avantage d'ôter tout caractère redondant aux deux expressions qui caractérisent le geste de la déesse, les

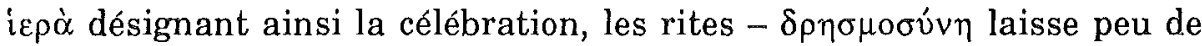
doute à cet égard - et öprı signifiant les objets mêmes de la révélation, ce qui est montré aux fidèles et qui est au point culminant de la célébration. Soulignons également que c'est la divinité elle-même qui fonde son propre culte, au sein d'une famille aristocratique, en l'accompagnant d'un interdit triplement formulé : on ne peut transgresser, violer

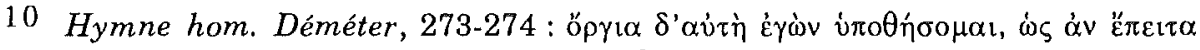

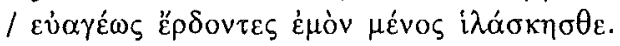

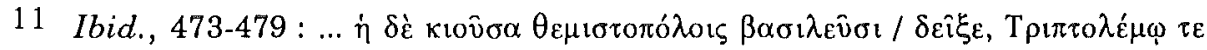

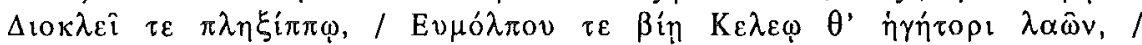

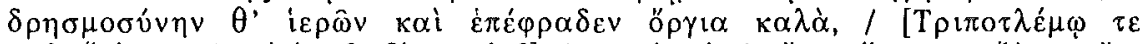

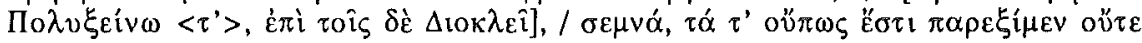

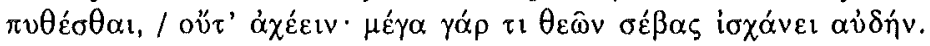


les óp $\gamma\llcorner\alpha$, ils ne peuvent être révélés, et l'on ne peut les connaître en s'informant ${ }^{12}$. Le verbe $\pi v \theta \varepsilon ́ \sigma \theta \alpha \imath$ sous-entend que l'expérience est le seul mode d'appréhension possible. Deux vers plus loin, le verbe ö $\pi \omega \pi \varepsilon v^{13}$ met l'accent sur le caractère visuel de la révélation, ce qui conforte l'hypothèse de la signification matérielle du mot ópyı.

L'importance bien connue de la vision dans les mystères des deux déesses est rapidement évoquée dans l'Héraclès d'Euripide ${ }^{14}$. Pour se rendre dans l'au-delà et en ramener Cerbère, le héros a "vu les ö $\rho \gamma \iota \alpha$. L'allusion aux mystères d'Éleusis est peu douteuse et renforce l'hypothèse que nous venons d'émettre.

Aristophane a, lui aussi, évoqué les őprı de Déméter. Dans les Grenouilles, le chœur des mystes aux Enfers répond à l'injonction du Coryphée d'entonner un hymne en l'honneur de la Basileia karpo-

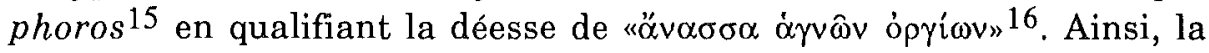
reine des fruits de la terre, célébrée par des danses dans les replis fleuris des prairies, est dite en même temps la maîtresse des saints ö $\gamma i \alpha$. La tentation est grande de rapprocher ces deux formulations, qui évoquent les «règnes" de la déesse et de mettre en relation, fût-elle symbolique, les óp $\gamma \iota \alpha$ dont elle est l'öv $\alpha \sigma \sigma \alpha$ et les produits de la terre, dont elle est la reine. Ainsi trouverait-on à nouveau dans cet emploi de öp $\gamma 1 \alpha$ une référence aux objets de la révélation initiatique ${ }^{17}$.

Mais les Mystères d'Éleusis ne sont pas seuls concernés par les öpria dans le culte de Déméter. Le même Aristophane, dans les Thesmophoriazousai, offre l'image caricaturale des femmes athéniennes en pleine dévotion. Rassemblées en un chœur de fidèles prêtes à la danse,

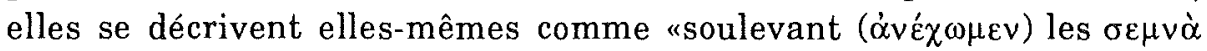
öpriø" en ces moments sacrés de la fête des Thesmophories ${ }^{18}$. Point de

12 Ibid., 478-479, vers cités à la note précédente.

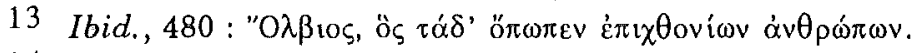

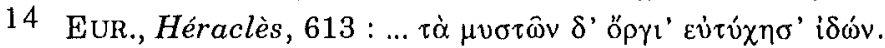

15 ARIsTOPH., Gren., 383.

16 Ibid., 384.

17 Un texte d'HipPocRATe évoque la beauté de l'initiation aux mystères et aux

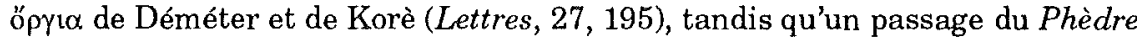

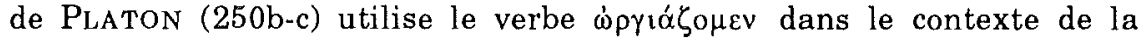
révélation du Beau par excellence, "vision bienheureuse», ce qui évoque sûrement les Mystères d'Éleusis. Cf. Chr. RIEDwEG, Mysterienterminologie bei Platon, Philon und Klemens von Alexandrien, Berlin, 1986 (Untersuchungen zur antiken Literatur und Geschichte, 26), p. 54.

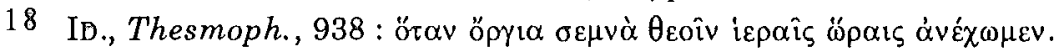


désignation générique de la célébration dans cet emploi d'ö $\rho \imath \imath$, mais plutôt le matériau faisant l'objet des manipulations sacralisantes. Une seconde fois dans l'œuvre, les ő $\gamma \gamma \downarrow$ des deux déesses sont évoquées par le chœur dans un contexte qui présente à trois reprises la notion d'ostentation et de vision :

“... les saints ö $\gamma \iota \alpha$ des deux déesses qu'il est interdit aux hommes de regarder, vous les montrez à la lueur des torches, vision immortelle..." 19

ce qui accentue encore l'idée d'objets concrets que suggère l'emploi du mot.

Hérodote connaît aussi des óp $\gamma \iota \alpha$ voués à Déméter. Cherchant à préciser l'origine des Géphyréens installés en Attique, il situe leur origine en Béotie et précise que, dans leur patrie d'adoption, ils ont des sanctuaires propres, en particulier un iepòv de Déméter Achaia et des öp $\gamma_{\imath} \alpha^{20}$. Plutarque explique l'épithète de la déesse en la mettant en

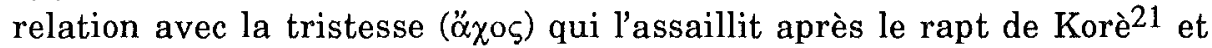
l'on sait que le culte de Déméter Achaia, assez répandu en Béotie, n'était pas sans rappeler la fête des Thesmophories ${ }^{22}$. C'est donc dans le même réseau de significations qu'apparaissent les ő $\rho$ ' $\alpha$ de Déméter, avec, dans ce cas précis, une marginalisation très claire de la célébration par rapport aux cultes athéniens. Cependant, il est difficile de se prononcer

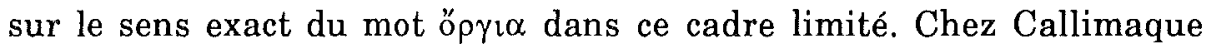
également, c'est dans le contexte, malheureusement fragmentaire, de la célébration des Thesmophories qu'apparaît le seul emploi d'őpri $\alpha$. À la suite d'événements qui nous échappent, la déesse est en colère, rapporte le poète, car «il n'est pas permis aux jeunes filles de voir les ő $\gamma$ ¡ $\alpha$ de Déo Thesmophoros» 23 .

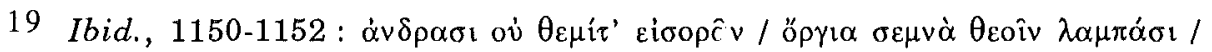

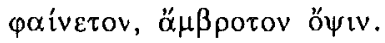

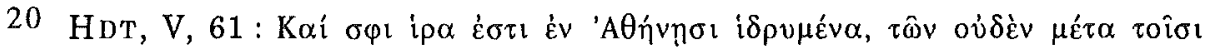

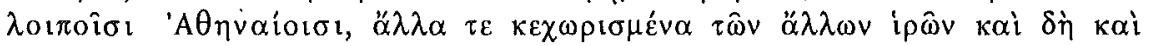

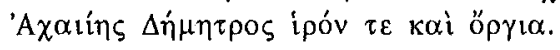

21 Plu'T., Is. et Os., 69 (Mor., 378d-e).

22 A. Schachter, Cults of Boiotia. 1. Acheloos to Hera, London, 1981 (BICS Suppl., 38.1), p. 159-160.

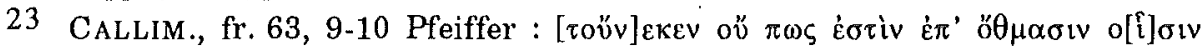

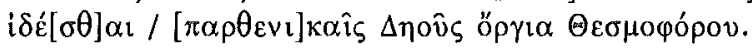


Le caractère originellement naturaliste des célébrations en l'honneur de Déméter, que ce soient les Mystères éleusiniens ou les Thesmophories, ne fait aucun doute. Lors des Thesmophories, dont le déroulement est mieux connu ${ }^{24}$, la manipulation d'objets directement liés aux ressources fécondantes de la nature constituait un des moments forts de la fête. Une scholie à Lucien confond, de façon significative, cette fête de Déméter, les Arrhéphories et les Skirophories ${ }^{25}$, trois célébrations qui s'articulaient autour du transfert et de l'utilisation de symboles de fécondité et de fertilité ${ }^{26}$. Les textes étudiés en relation avec les őp désigner ces objets, dont la nature appelle le secret. Tel est bien le sens auquel il convient de penser prioritairement dans la plupart des emplois rencontrés jusqu'ici. Néanmoins, dans de nombreux cas ultérieurs, ǒ $p \gamma \downarrow \alpha$ désigne très clairement une célébration, un ensemble particulier d'actions rituelles vouées aux déesses ${ }^{27}$. On peut dès lors considérer que ǒ $\rho \gamma \imath \alpha$, dans ces emplois, relève de la désignation par synecdoque. Autrement dit, le nom de l'élément central du rituel - l'objet même de la révélation - s'est étendu à toute la cérémonie ${ }^{28}$.

24 L. Deubner, Attische Feste, Hildesheim, $1969^{2}$ [1932], p. 50-60; M. Detienne, Violentes «eugénies». En pleines Thesmophories, des femmes couvertes de sang, in La cuisine du sacrifice, op. cit. (n. 3), p. 192-193; A.C. BRUMFIELD, The Attic Festivals of Demeter and their Relation to the Agricultural Year, Salem, 1981, p. 70-79; W. BurkerT, Homo Necans. The Anthropology of Ancient Greek Sacrificial Ritual and Myth, Berkeley, 1983 [or. all. 1972], p. 256-259; ID., Greek Religion, Harvard, 1985 [or. all. 1977], p. 242-243.

25 Cf. A.C. Blumfield, op. cit., p. 74-79, 98; P. Brule, La fille d'Athènes. La religion des filles à Athènes à l'époque classique. Mythes, cultes, société, Paris, 1987, p. 82.

26 Ces fêtes avaient également une dimension politique non négligeable, mais qui n'ôte rien au substrat agraire de leur célébration.

27 Par exemple, Hippocrate, Lettres, XXVII, 195; Plut, fr. 24, cité par Hippolyte, Refutatio, V, 20, 5; PAUS., IV, 1, 7; IV, 15, 7.

28 Cf. C. CALAME, art. cit., p. 198-200, évoque toute une série de désignations par

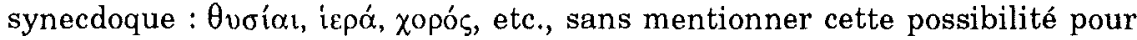

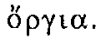




\section{Dionysos et ses ǒpүra}

Si le dieu n'a pas la priorité chronologique dans la documentation, il s'y taille pourtant la part la plus importante. Le lien le plus ancien entre Dionysos et les ő $p \gamma$ i est opéré par Hérodote, dans un bref passage sur l'interdit de la laine dans les funérailles égyptiennes, interdit que l'on retrouve dans les prescriptions imposées aux initiés des óp pythagoriciennes en relation avec Dionysos Zagreus ${ }^{29}$. Mais c'est

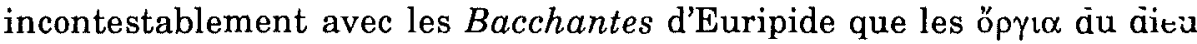
entrent par la grande porte dar c la littérature, et par là même dans notre connaissance de ces pratiques religieuses. Le Tragique nous apprend

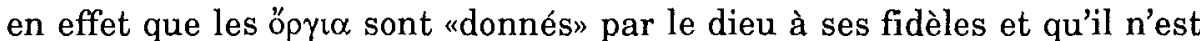
pas permis aux non-initiés d'en connaitre la nature ${ }^{30}$; il évoque encore le caractère nocturne et choral des célébrations $\mathrm{s}^{31}$ dans le cadre privilégié de la montagne ${ }^{32}$, de même que l'habillement spécifique des sectateurs du dieu qui, en l'occurrence, sont en majeure partie des femmes ${ }^{33}$. C'est également avec Euripide qu'apparaissent pour la première fois les óp tions en l'honneur de Dionysos. Strabon ne manquera pas de citer ce passage lorsqu'il voudra prouver la parenté entre les divers types de Kourètes serviteurs des dieux en soulignant le lien étroit entre le culte de la Mère des dieux parmi les Phrygiens et celui de Dionysos chez les Grecs $^{34}$. Cette mention prouve assez que, dès l'Antiquité, la tragédie d'Euripide servait de référence en ces matières.

Néanmoins, il faut attendre la 26e Idylle de Théocrite pour voir réapparaître les ö $\rho \gamma \alpha$ du dieu avec une évocation de leur nature. Penthée s'est dissimulé pour observer le cérémonial bachique célébré par sa mère et ses tantes. Après avoir cueilli divers végétaux dans une "prairie pure», elles y dressent trois autels pour Sémélé et neuf pour Dionysos. De corbeilles elles extraient des gâteaux sacrés qu'elles

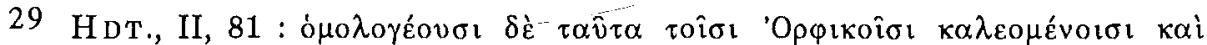

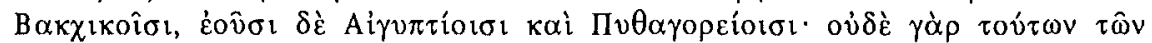

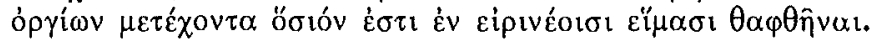

30 EUR., Bacch., 472 : ó

31 Ibid., 486. Apollonios de Rhodes, Argon., II, 907, décrivant les mystères

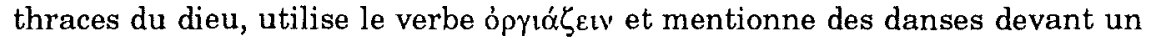
antre et un cérémonial nocturne.

32 Eur., Bacch., 191.

33 Ibid., 827-836.

34 STRABON, X, 3, 13 (C469). 
disposent sur les autels de verdure, ainsi que l'a enseigné le dieu luimême. Apercevant Penthée, une des Bacchantes entre dans une colère noire et "renverse du pied les ö $\gamma 1 \alpha$ de Bacchos qui rend fou» ${ }^{35}$ dans le

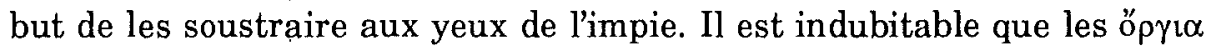
du dieu sont, dans ce cas précis, les gâteaux extraits des cistes ${ }^{36}$. Dès

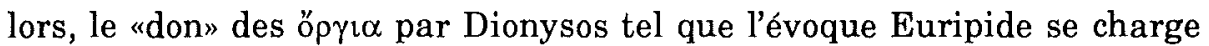
de l'ambiguïté déjà énoncée à propos des ő $\rho \gamma \imath \alpha$ de Déméter et peut concerner tant les objets que leur mise en œuvre, même s'il faut souligner qu'aucune mention d'objets, hormis les thyrses et le vêtement dionysiaque, n'apparaît dans la tragédie.

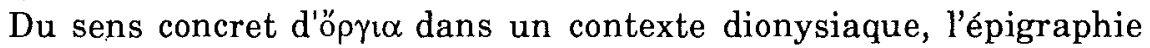
donne un exemple bien mis en lumière par Albert Henrichs qui a montré que l'épigramme funéraire d'une ménade milésienne, datée du IIIe-IIe siècle av. J.-C. et portant la mention d'ôp $\gamma \iota \alpha$, faisait référence à des objets emmenés sur la montagne et non pas à des actes cultuels ${ }^{37}$. Il n'en demeure pas moins qu'un règlement religieux du culte de Dionysos provenant également de Milet et daté de l'année 276/5 avant notre ère, utilise óp $\gamma \iota \alpha$ comme terme générique des célébrations en l'honneur du dieu ${ }^{38}$. Un oracle delphique donné aux habitants de Magnésie du Méandre fait quant à lui surgir l'ambiguité en évoquant, à la manière d'Euripide, le don des őpyı et des bonnes lois de Dionysos à la cité par trois ménades thébaines ${ }^{39}$.

Différents textes plus tardifs permettent également d'éclairer la signification et la portée des őpyı de Dionysos. Un passage du De dea Syria de Lucien décrivant le sanctuaire de la déesse à Hiérapolis évoque l'óp $\gamma$ iov de Dionysos qui s'avère être un petit bronze ithyphal-

35 THEOCR., Id., XXVI, 13.

36 Cf. A.S.F. Gow, Theocritus, II, Commentary, Cambridge, 1952, p. 479; A. Henrichs, Die Maenaden von Milet, in ZPE, 4 (1969), p. 227-229.

37 A. HENRICHs, art. cit., p. 223-241.

38 F. Sokolowski, LSAM, Paris, 1955, n 48. Cf. A. HenRICHS, art. cit., p. 236. Cela semble être également le cas dans une inscription de Téos datée du Ier siècle avant notre ère : $S E G, I V, 598$.

39 O. KeRN, Die Inschriften von Magnesien am Meander, $n^{\circ} 215$ (mi-Ier siècle ap. J.-C.). Cf. H.W. PARKe \& D.E.W. Wormelu, The Delphic Oracle, I : The History, Oxford, 1956, p. 331-332 (et t. II, $n^{\circ} 338$ ). - En matière d'inscriptions en l'honneur de Dionysos, il faut aussi mentionner le péan delphique de Philodamos, daté des environs de 340 av. J.-C. qui célèbre "le Iacchos des saints ópyı que chante le peuple de toute la terre de Grèce lors des révélations..."

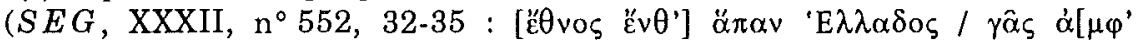

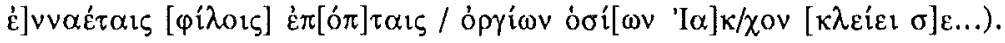


lique $^{40}$, tandis que Clément d'Alexandrie, dans le souci apologétique de démystifier les corbeilles sacrées, décrit leur contenu : «des gâteaux de sésame et de miel, du miel et de la farine, des gâteaux en pelote, des gâteaux à bosses, des grains de sel, et un serpent, ö $\rho \gamma$ ıov de Dionysos Bassaros ${ }^{41}$. Mis à part un hymne orphique en l'honneur du dieu ${ }^{42}$, ce sont les deux seuls emplois du terme au singulier dans un cadre dionysiaque, et ils fournissent une clé d'interprétation des objets cultuels, car l'ópyıov cité par Clément en apposition à $\delta \rho \alpha \kappa \hat{\omega} v$ renvoie aux ő $\rho \gamma\llcorner\alpha$ extraits des corbeilles par les Bacchantes de Théocrite. Le symbolisme chthonien attaché à l'image du serpent s'insère adéquatement dans un rituel dont on pressent la relation intime avec les forces de vie dans toute leur exubérance, qu'elles soient végétales ou humaines, comme en atteste la statuette au sexe disproportionné décrite par Lucien ${ }^{43}$.

\section{Hécate et les Grands dieux de Samothrace}

Dans son développement célèbre sur l'origine égyptienne des coutumes religieuses grecques, Hérodote fait quelques exceptions, dont une concerne la représentation ithyphallique des hermès ${ }^{44}$. Cette convention iconographique trouverait, selon lui, sa source chez les Athéniens qui l'auraient empruntée aux Pélasges venus habiter avec eux avant d'aller s'installer à Samothrace. Et Hérodote d'enchaîner, "celui qui est initié aux öpyı des Kabires que célèbrent les Samothraciens pour l'avoir appris des Pélasges sait ce que je veux dire». Et une ébauche d'explication s'amorce ensuite : "ces Pélasges qui avaient habité avec les Athéniens habitaient en effet jadis Samothrace, et c'est d'eux que les Samothraciens tiennent les óp $\gamma(\alpha$. Quant au fait de représenter les statues d'Hermès avec un sexe, les Athéniens furent les premiers des Grecs à l'avoir appris des Pélasges. Et les Pélasges rapportaient un récit sacré à ce propos, ce qui était montré dans les mystères de Samothrace» ${ }^{45}$. Cette dernière proposition est introduite par

\section{LUCIEn, De Dea Syria, 16.}

41 Clem. Alex., Protr., II, 22, 4., cité comme tel par Eusebe, Prép. évang., II, 3, 39 :

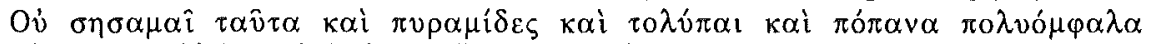

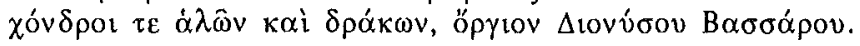

42 Hymnes orphiques, $52,5$.

43 Quel que soit le degré de véracité du récit de Lucien, il n'en reste pas moins que l'image du bronze ithyphallique fait surgir le mot öprov.

44 HDT., II, 51.

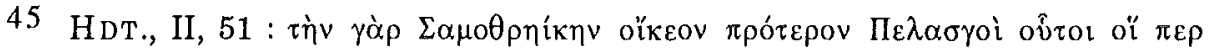

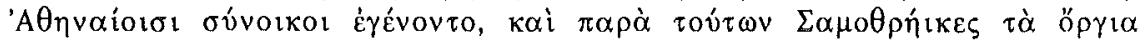


le relatif $\tau \grave{\alpha}$; or aucun neutre pluriel n'apparaît dans la principale. Le relatif renvoie donc à un mot cité antérieurement. Deux neutres pluriels peuvent être invoqués : $\tau \dot{\alpha} \dot{\alpha} \gamma \alpha \dot{\alpha} \lambda \mu \alpha \tau \alpha$ ou $\tau \dot{\alpha}$ ő $\rho \gamma$ i $\alpha$. Vu que les statues apparaissent dans la phrase précédente, c'est vraisemblablement elles qui étaient «montrées» lors des mystères. Compte tenu du sens ambigu d'ópyı $\alpha$ tel qu'il a été mis en évidence jusqu'à présent, on peut supposer que les óp $\gamma \propto \alpha$ des Kabires présentés confusément par Hérodote faisaient tout autant référence aux objets qu'à la fête qui les mettait en scène. Quoi qu'il en soit, le symbolisme sexuel semble patent et le texte de l'historien insère cette célébration dans un même registre d'exaltation des forces fécondes que dans les cas analysés précédemment.

Néanmoins, les mystères des Kabires peuvent rencontrer d'autres aspirations. Les Grands dieux de Samothrace étaient notamment des protecteurs de la navigation et c'est en recevant leur initiation que les Argonautes obtiennent les secrets pour arriver à bon port. Sous la plume d'Apollonios de Rhodes, les Grands dieux sont les détenteurs d'ópyla qu'il n'est pas permis de chanter ${ }^{46}$. Une autre divinité est chez cet auteur mise en relation avec les öp $\gamma\llcorner\alpha$ : c'est Hécate, la déesse protectrice des magiciennes qu'invoque Médée en jurant «par les ő $\rho$ i $\alpha$ de la vierge coureuse des nuits, la fille de Persès 47 ".

\section{Aphrodite et les Muses}

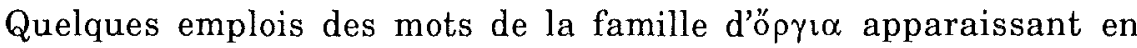
relation avec des divinités ne font pas référence à un culte proprement dit. Elles méritent donc un traitement séparé.

Pour les époques qui nous occupent, ce sont les comédies d'Aristophane qui offrent la majeure partie de ces emplois. Dans la Lysistrata, le poète utilise à deux reprises un terme de cette famille et chaque fois en relation avec Aphrodite. Il s'agit d'abord de la description métaphorique de l'état dans lequel se présente un des maris privé de sa

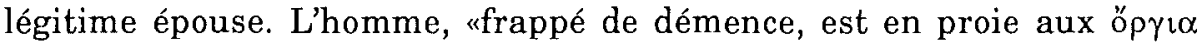
d'Aphrodite $^{48}$ ". Et le mari de reprocher à sa femme, un peu plus loin, de

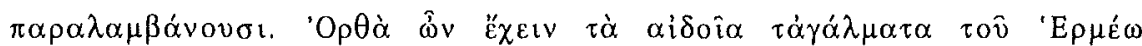

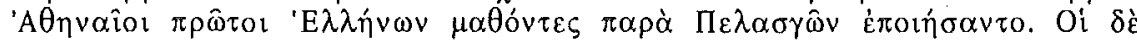

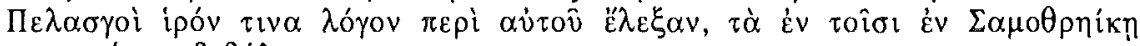
$\mu \nu \sigma \tau \eta \rho i o \imath \sigma \imath \delta \varepsilon \delta \dot{\eta} \lambda \omega \tau \alpha \iota$

46 APOLL. RHODES, Argon., I, 920.

47 Ibid, IV, 1020.

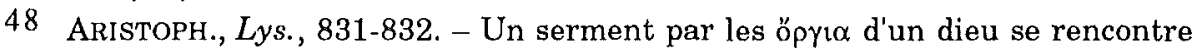
dans une inscription de Thessalonique datée du Ier siècle de notre ère (SEG, $\mathrm{XXX}, 622$ avec la bibliographie antérieure). 
n'avoir plus pratiqué depuis longtemps les iepó de la déesse; le mot qu:

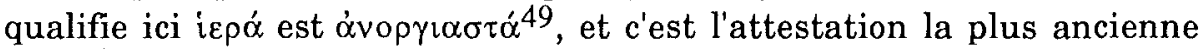
de cet adjectif.

Van den Burgh dénie à ces exemples toute connotation religieuse en les reléguant parmi les métaphores. Tout en reconnaissant l'absence de référence cultuelle proprement dite, il nous semble maladroit de ne pas chercher à tirer parti de tels emplois pour la compréhension des ó $\rho \gamma$ i $\alpha$. En effet, une image n'a jamais autant de force qu'en se référant directement au sens premier de la réalité dont elle joue. Or le poète, en mettant en relation une divinité donnée - en l'occurrence Aphrodite - et une notion sortie en droite ligne du vocabulaire religieux, sollicitait la compréhension et le rire de son public. Cette préoccupation, que suppose l'association d'Aphrodite et des óp $\gamma\llcorner\alpha$, laisse entendre que la sexualité que patronne la déesse, avec les relations qu'implique un tel domaine à la nuit, au secret et à la fécondité, n'était pas sans rapport avec la notion même d'ő $\rho$ i $\alpha$, particulièrement bien adaptée au propos d'Aristophane. Cet emploi mettant Aphrodite, tout comme Éros, en rapport avec les ö $\gamma \_\alpha$ se multipliera au fil du temps ${ }^{50}$.

Quant aux Muses, elles se voient également attribuer des ő $\gamma 1 \alpha$ toutes métaphoriques, dont on trouve un premier exemple dans les Grenouilles $^{51}$. L'aspect choral et musical, peu souligné jusqu'à présent mais fondamental dans la célébration de certains ő $\rho$ l $\alpha$, justifierait à lui seul cette association. Mais les Muses sont aussi des divinités inspiratrices, qui insufflent le divin au cœur de l'homme et surgissent d'un paysage luxuriant pour inspirer les poètes. De telles considérations contribuent elles aussi à éclairer la veine religieuse à laquelle appartiennent les ő $\gamma$ $\alpha$.

49 Ibid., 898.

50 Anth. Pal., VI, 80; VII, 222; IX, 220; X, 21; Clem. AleX., Protr., II, 13, 4; Eus., Prép. évang., II, 3, 12; NonNos, Dion., XLII, 373.

51 ARISTOPH., Gren., 356. 


\section{De quelques emplois du sens prétendument général}

Quittant à présent le sentier balisé du culte des divinités particulières, il convient d'examiner quelques textes tragiques appelés à la

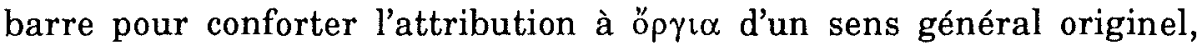
déduit de l'étymologie supposée du mot.

Dans Les Sept contre Thèbes d'Eschyle, le chœur supplie les dieux de préserver la cité de l'attaque argienne en les invoquant par leurs noms, les uns à. la suite des autres, et rappelle les cérémonies, les sacrifices célébrés par la cité : "souvenez-vous des iєpâv $\delta \eta \mu \iota \hat{v}$, souvenez-vous

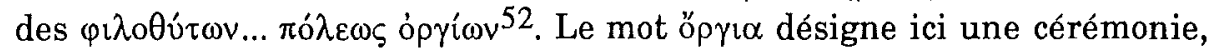
compte tenu de l'adjectif qui le qualifie, "prodigue en sacrifices»; soit il s'agit d'une redondance par rapport à ípó, ce qui lui ôte tout caractère spécifique, soit il s'agit d'un autre type de cérémonie, ce que l'on croira plus volontiers. Or, parmi les divinités du panthéon thébain invoquées, un dieu et une déesse manquent à l'appel, Dionysos et Déméter, dont le culte thébain était connu des auteurs tragiques, tout au moins dans le cas de Dionysos. Ne pourrait-on imaginer que les ő $\gamma \iota \alpha$ dont doivent se souvenir les puissants de l'Olympe intègrent, mais indirectement, ces deux divinités à la prière désespérée du chœur? L'hypothèse est audacieuse, reconnaissons-le, mais trouve un fondement dans l'intime relation entre le nom d'ő $p \gamma \alpha$ et ces deux grands absents du panthéon.

Sophocle use du terme à deux reprises dans la partie de son œuvre qui nous est conservée. Dans Les Trachiniennes, Héraclès revenant d'une campagne guerrière s'apprête à offrir un sacrifice à Zeus lorsque le héraut lui apporte le présent de Déjanire, la tunique mortelle, dont il s'enveloppe aussitôt. Alors, la flamme des $\sigma \varepsilon \mu v \omega \hat{v} v$ ó $\gamma$ í $\omega v$ tarde à paraître, les effets du vêtement empoisonné commencent à se faire sentir et Héraclès sombre dans la douleur et dans la folie ${ }^{53}$. "O $\rho \gamma\llcorner\alpha$, dans ce cas, soit désigne "l'offrande" en simple synonyme de ípó, soit possède une connotation particulière que fournit peut-être le contexte. Avant de trancher, soulignons le sens concret d'öp $p$ i $\alpha$ dans cet emploi et voyons le contexte.

Le sacrifice qu'Héraclès célèbre sort de la normalité puisque la flamme bien droite, heureux présage d'un sacrifice efficace, refuse de s'allumer et que l'action de grâce s'achève par la folie du héros. Bien qu'il soit difficile, voire impossible, de préciser la connotation exacte

52 Esch., Sept, 179.

53 SopH., Trach., 765. 


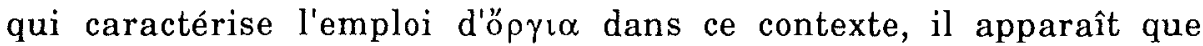
l'offrande ainsi désignée sort de la normalité. De même dans l'Antigone, lorsque Tirésias entend convaincre Créon d'offrir une sépulture à Polynice en lui décrivant tous les signes négatifs que lui envoient les dieux, et notamment l'absence de flamme sur les autels à feu, il conclut en insistant sur «les présages caduques d'ő $p \gamma(\alpha$ qui n'ont aucun sens $\mathrm{s}^{54}$. Dans un cas comme dans l'autre, le sacrifice intègre la notion d'öp $\gamma$ co dans une situation extrême, lorsque la cérémonie tourne mal, lorsque l'inconscience des hommes (celle de Créon, celle de Déjanire) transgresse un ordre établi et apporte le meurtre, la folie ou la

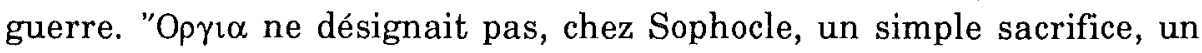
acte cultuel ordinaire, mais l'on dispose malheureusement de trop peu d'occurrences pour se prononcer plus fermement.

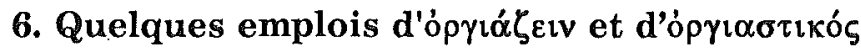

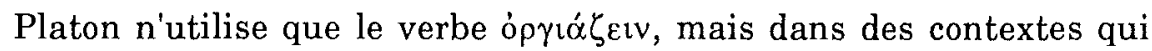
sont loin d'être indifférents. Dans le Phèdre (252d), tout d'abord, il affirme que l'être aimé représente pour l'amant la divinité elle-même, "c'est comme une sorte d'image sainte qu'il se fabrique et qu'il orne

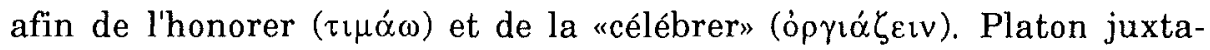

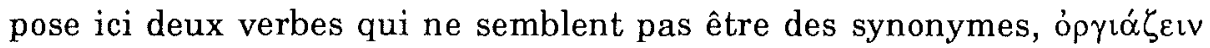

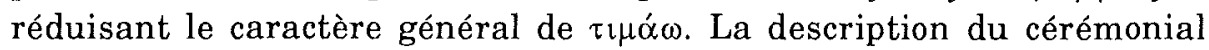
que célèbre l'amant relève d'une métaphore qui est censée illustrer l'aspiration à une intimité avec la divinité projetée sur l'aimé terrestre. Dès lors, compte tenu du sens très large de $\tau \imath \mu \alpha ́ \alpha \omega$, on peut faire

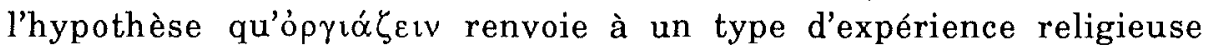
correspondant précisément à une telle recherche de communion.

Dans un passage des Lois, Platon souligne combien il est nécessaire d'honorer les dieux; il présente à cette fin une hiérarchie systématique des catégories divines qu'il convient de célébrer. Après les Olympiens et les divinités chthoniennes, ce sont les démons et les héros qu'il importe aussi d'honorer, ó $\rho \gamma \dot{\alpha} \alpha \zeta_{\varepsilon l v}{ }^{55}$. Rappelons que Platon accorde un statut particulier aux démons dans certains dialogues en les érigeant en intermédiaires entre les dieux et les hommes. Plutarque, d'autre part, faisant écho à cette conception, introduit également la notion d'óp $\gamma\llcorner\alpha$ : les tâches des démons serviteurs des dieux est notamment de concélé-

54 ID., Ant., 1013.

55 Platon, Lois, IV, 717b. 


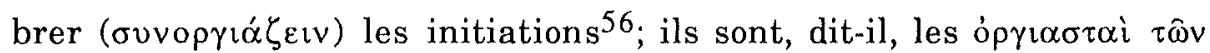
$\tau \varepsilon \lambda \varepsilon \tau \omega{ }^{57}$. Peut-être l'expérience religieuse que suscitaient les öp $\gamma \iota \alpha$ correspondait-elle plus particulièrement au culte de certains démons 58 .

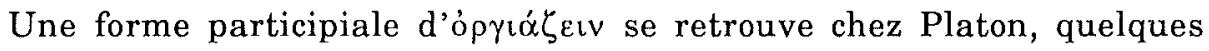
lignes plus loin, dans le cadre des cérémonies privées à accorder aux dieux Patrôoi ${ }^{59}$. Ce deuxième emploi illustre le caractère privé que peuvent revêtir les öp $\gamma$ ı, signification que l'on retrouve dans un autre passage des Lois où le philosophe interdit l'établissement et la célébra-

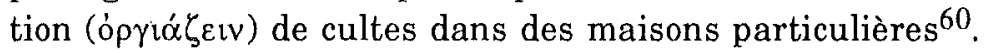

Chez Aristote, la notion est davantage précisée. L'adjectif

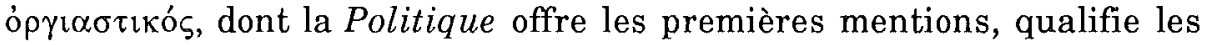
transports que suscite la musique de l'aulos dont le philosophepédagogue déconseille l'apprentissage. Un tel instrument n'a en effet rien de moral ${ }^{61}$, mais fait entrer le musicien - et son auditoire - dans un état $\pi \alpha \theta \eta \tau \iota \kappa o ́ \varsigma^{62}$. C'est dans la même perspective qu'apparaît le verbe

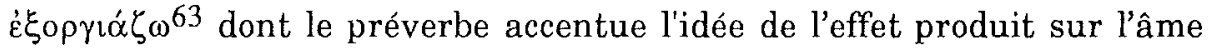
par l'action de la musique. La catégorie "orgiastique» ainsi définie par Aristote se réfère à un type d'expérience qui implique une réaction émotionnelle des participants et un investissement affectif. Transposée sur un plan religieux, l'utilisation des mots de cette famille fait indubitablement surgir une même catégorie d'actions et de réactions, confirmant que l'élément «orgiastique» constitue une expérience religieuse originale et marginale.

Ce caractère marginal semble trouver une confirmation dans le silence des orateurs sur le sujet. On sait que Jean Rudhardt, dans sa thèse sur les Notions fondamentales et actes constitutifs du culte s'est essentiellement fondé sur le corpus fourni par les discours du IVe

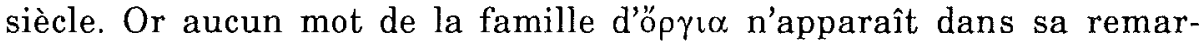
quable analyse du vocabulaire religieux. Il existe en réalité deux occurrences. La première remonte à l'Aréopagitique ; évoquant la rigueur et

56 PLuT., La face de la Lune, 944c-d.

57 Plur., De la disparition des oracles, 13 (Mor., 417a).

58 Un passage de l'Epinomis (985d) fait apparaître l'adjectif óvop signifier que certains dieux "naturels» ne reçoivent pas les honneurs qu'ils méritent.

59 Ibid.

60 Platon, Lois, X, 910b-c.

61 ARISTOTE, Pol., VIII, 6, 9 (1341a).

62 Ibid, VIII, 7, 9 (1342b).

63 Ibid., VIII, 7, 4(1342a). 
l'ordre qui présidaient à la direction des affaires de la cité au temps passé, Isocrate souligne la régularité avec laquelle on servait

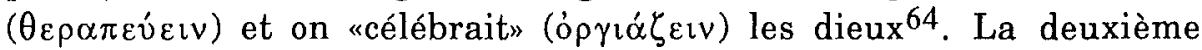
occurrence apparaît dans un fragment de Dinarque sur les orgéons qu'il définit comme «ceux qui célèbrent (ó $\gamma \gamma$ tó $\zeta \varepsilon ı v)$ les dieux établis en privén ${ }^{65}$, usant donc des mêmes termes que Platon lorsqu'il interdisait les manifestations cultuelles dans des maisons particulières. On peut

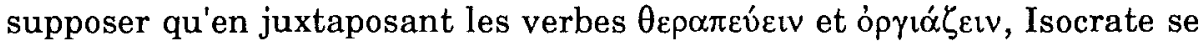
réfère à une même distinction que Platon entre $\tau \iota \mu \alpha \dot{\alpha} \omega$ et óp $\gamma \downarrow \dot{\alpha} \zeta \omega$, le second verbe désignant un autre type d'activité cultuelle, d'un caractère plus privé.

Le fragment de Dinarque pose deux questions : qu'entend-on exactement par orgéons, et quelle est la relation entre ö $\rho \gamma 1 \alpha$ et ó $\rho \gamma \varepsilon \omega^{6}{ }^{66}$ ? Il est bien entendu exclu dans le cadre de cette étude de proposer une vision d'ensemble du statut des orgéons. On tentera seulement de trouver dans une brève approche de la nature de leur association un éclairage supplémentaire pour la définition des óp $\gamma\llcorner\alpha$.

Deux textes intéressent directement notre propos. Un passage de l'Éthique à Eudème d'Aristote, évoquant les diverses "communautés" intégrées dans la cité, juxtapose les associations des phratères, celles

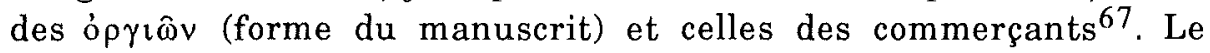

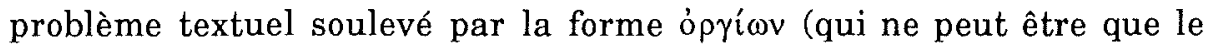
génitif pluriel de ő $\rho \gamma(\alpha)$ a conduit les éditeurs à lui substituer ó $\gamma \varepsilon \omega v \hat{\omega} v$, plus satisfaisant pour la compréhension du texte. Cependant, dans un fragment d'une loi attribuée à Solon par le juriste romain Gaius, on retrouve le même problème textuel : dans une énumération de groupe-

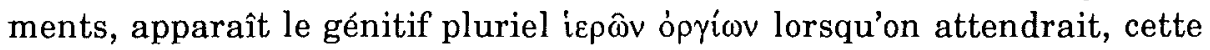

64 IsOCRATE, Aérop. (VII), 29.

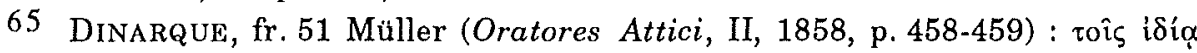

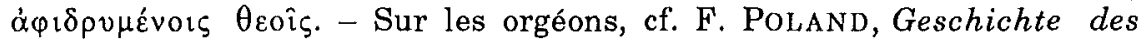
griechischen Vereinswesens, Leipzig, 1909, p. 12-13; W.S. FERGUSON, The Attic orgeones, in HThR, 37 (1944), p. 62-140; J. VELISSAROPOULOS, Les nauclères grecs. Recherches sur les institutions maritimes en Grèce et dans l'Orient hellénisé, Genève-Paris, 1980, p. 94-95.

66 L'Hymne ps.-hom. à Apollon (389) fait apparaitre un hapax, manifestement en relation avec cette famille de mots puisque l'auteur désigne les prêtres que recherche le dieu pour honorer son culte delphique sous le nom d'óprioves. Cf. M. MeIER-BRUGGER, art. cit., p. 105-106.

67 ARISTOTE, Ethique à Eudème, VII, 9, 3 (1241b). 
fois, un nominatif pluriel mentionnant un groupe d'individus ${ }^{68}$. Certains ont proposé d'ajouter un mot au nominatif pluriel que

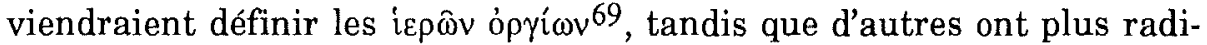

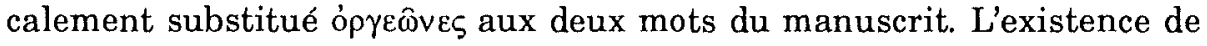
ces deux problèmes philologiques, causés par l'apparition d'ôpyı là où l'on attendrait ó $\rho \varepsilon^{\prime} \omega v$, permet de supposer que, aux époques archaïque et

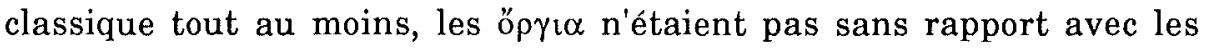
orgéons. Or la liaison étroite entre les phratères et les orgéons dans ces textes et dans d'autres ${ }^{70}$ donne à penser que le terme "orgéon" servait à désigner les membres de groupements dans leur activité religieuse, c'est-à-dire en tant que sectateurs du ou des même(s) dieu(s) ou héros, ce qui viendrait confirmer le caractère plus "privé» des cultes évoqués par le verbe ó $\rho \gamma \dot{\alpha} \zeta \varepsilon \imath v$ chez Platon et Isocrate ${ }^{71}$.

\section{7. Éléments de conclusion}

Revenons à présent aux questions énoncées dans l'introduction afin d'essayer de préciser les signifiés que véhiculent ópyı et les mots de sa famille.

La question de l'étymologie reste ouverte, dans la mesure où le sens prétendument général du mot "ce qui est accompli, acte cultuel en

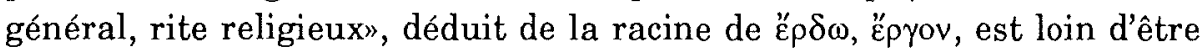
conforté par les textes dont nous disposons. Il est curieux de lire chez certains auteurs modernes que les Anciens lexicographes avaient "oublié" le sens originel du mot puisqu'ils expliquaient le mot en recou-

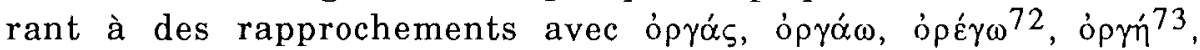
öркоร ${ }^{74}$; nous dirons plutôt qu'ils ne l'avaient jamais su, et que les expli-

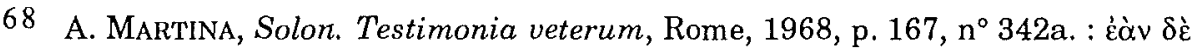

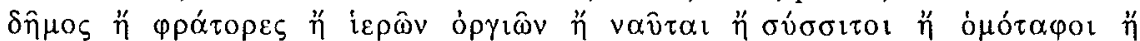

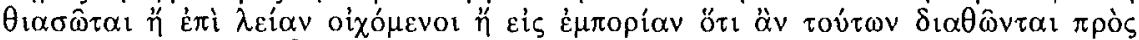

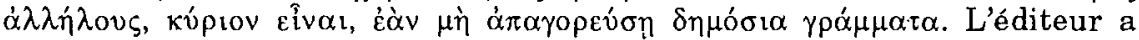

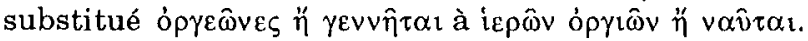

69 Ibid., dans l'apparat critique.

70 Cf. W.S. FERGUSON, art. cit.

71 À l'époque hellénistique, les associations d'orgéons se multiplient, notamment pour célébrer le culte de divinités importées; elles perdent dès lors leur caractère "familial», tout en conservant un caractère privé, que l'on peut même taxer de marginal.

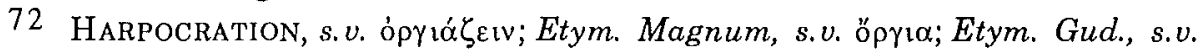
öp $\gamma 1 \alpha$.

73 Clem. Alex., Protr., II, 13, 1; Servius, Ad AEn., IV, 302.

74 Anecdota Oxoniensis, II, 307 Cramer. 
cations données en disent plus long sur la signification qu'ils attribuaient à cette famille de mots que l'étymologie la plus savante, quelle qu'en soit la pertinence.

Dès les premières attestations, nous avons constaté qu'óp $\gamma(\alpha$ pouvait autant concerner un objet qu'une cérémonie, ce que des exemples ultérieurs n'ont fait que confirmer. Il est dès lors probable, comme nous l'avions déjà laissé entendre, que les objets cultuels au cœur du mystère sacré ont donné leur nom à l'ensemble de la célébration; cette ambivalence est très claire dans un passage de Plutarque décrivant une matrone romaine qui fait cesser - ce qui implique une célébration - et voiler - ce qui suppose des objets - les óp $\gamma^{\prime} \alpha^{75}$, lorsqu'un homme est découvert au cœur d'une fête de femmes. Cette interprétation permet

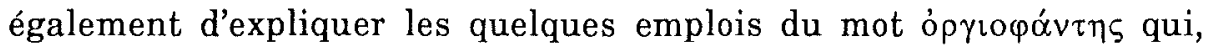

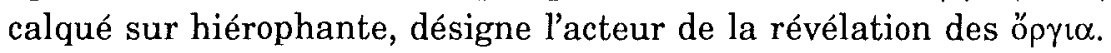

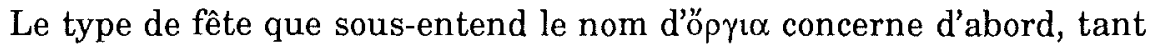
dans une perspective chronologique que quantitative, Déméter et Dionysos, la Mère des dieux - qu'on l'appelle Rhéa, Cybèle ou Grande Mère -, les Grands dieux de Samothrace, qu'ils soient ou non identifiés aux Kabires - et Hécate. À partir de l'époque hellénistique apparaissent également, mais dans une moindre mesure, Adonis, Baal, Hélios, Isis, Osiris, Mithra, Palaimon et le Zeus crétois. Une attestation très isolée, due à Plutarque, qualifie d'ó $\rho \gamma \imath \alpha$ les Plyntéries athéniennes en

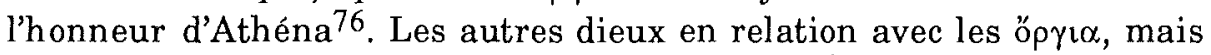
sans référence directe à un culte, sont Aphrodite, Éros et les Muses.

Le caractère naturaliste de la grande majorité des rites laisse penser que certains de ces cultes avaient, du moins à l'origine, un rapport direct avec la croissance des espèces végétales ${ }^{77}$ et plusieurs textes attestent le symbolisme sexuel des objets frappés d'interdit et révélés aux seuls initiés. Quelques exemples plus tardifs établissent un

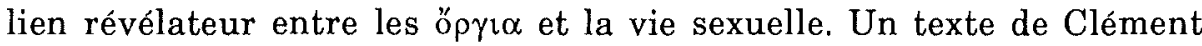
d'Alexandrie évoquant les relations conjugales, insiste sur la nécessité

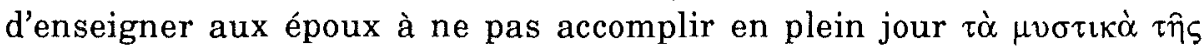
$\varphi v ́ \sigma \varepsilon \omega \varsigma$ ö $\rho \gamma 1 \alpha^{78}$, tandis que Nonnos, dans sa manière foisonnante de décanter les symboles, utilise ő $p$ ı $\alpha$ à maintes reprises pour désigner les

75 Plut., César, 10,3:

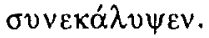

76 Cf. l'étude de M. Christopoulos dans ce même volume.

77 Cf. L. GERNET, op. cit., p. 125.

78 Clem. AleX., Pédagogue, II, 10, 96, 2. 
attraits charnels des superbes héroïnes de son épopée dionysiaque ${ }^{79}$, évoquant à la fois la sexualité et le mystère qui l'entoure.

Le lieu idéal de la célébration d'ô $\rho \gamma \imath \alpha$ est à la lisière des cités, en pleine nature ou même aux extrémités du monde - qu'on se souvienne seulement du désir des Bacchantes d'Euripide de célébrer leur dieu à Chypre, en Égypte ou sur les flancs de l'Olympe. Les interdits et le caractère impératif du secret en constituent des prescriptions fondamentales, impliquant une révélation procurée par une «monstration» dans la majeure partie des cas. Plus qu'un enseignement, l'initiation au mystère divin prend la forme d'une expérience qui sollicite d'abord le regard, mais également l'audition - la musique est une composante essentielle de l'expérience orgiastique et il est significatif que la seule

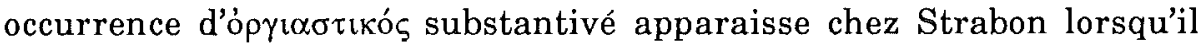
insiste sur l'importance de la musique dans la fête ${ }^{80}$.

Le temps idéal de la célébration, c'est la nuit, "car les ténèbres sont plus saintes», explique Dionysos dans les Bacchantes. Il va sans dire que toutes les célébrations ne se déroulent pas nécessairement pendant la nuit, mais l'obscurité et les ténèbres peuvent également désigner, sur un mode symbolique, le prélude à la révélation.

La place de la divinité célébrée par des óp dans le cas de Déméter et de Dionysos, car, non seulement elle a enseigné elle-même la nature et les prescriptions de son culte, mais elle $\mathrm{y}$ prend part très directement et l'expérience mystique du fidèle ne semble pas exclure une sorte de communion avec la divinité.

On comprend dès lors pourquoi certains auteurs chrétiens - ou juifs, comme Philon d'Alexandrie -, tout en stigmatisant les excès des païens, ont repris à leur compte un mot comme ópyı pour désigner certaines de leurs célébrations, notamment la notion de mystère sacré accompagné d'une communion avec la divinité.

Il apparaît, au terme de cette étude, que le mot ő $\rho \gamma \alpha$ ne peut être traduit de manière univoque, et que la prise en compte de la nature des sources et des contextes est indispensable pour rendre, dans une traduction, la portée du terme dans un texte donné. Bien souvent, il conviendrait de maintenir la forme grecque des mots, étant donné les connotations négatives du mot français "orgies» et la difficulté de rendre en traduction tout l'ambiguité que recèle une telle "désignation par

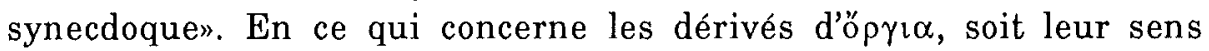

79 NoNNOS, VII, 266; XI, 506.

80 Strabon, X, 3, 10 (C468). Ce passage complexe mériterait une étude en soi car il s'agit de la première réflexion théorique sur le sujet. 
correspond aux éléments de définition que nous venons d'esquisser, soit - et l'on pense notamment à ó $\rho \gamma \imath \alpha ́ \zeta \omega$ chez Platon et Aristote - ils paraissent souligner avant tout le caractère privé, ou même marginal, de manifestations religieuses.

Il est évident que toutes les cérémonies comprenant quelques éléments constitutifs de ce type de célébration n'ont pas été appréhendées ici, étant donné la base lexicographique de l'exposé et l'existence d'autres familles de mots servant à le désigner ( $\mu \nu \sigma \tau \eta \dot{\eta} \rho \iota v$ par exemple ${ }^{81}$ ). Il apparaissait néanmoins indispensable de regarder d'un œil dégagé de tout a priori les emplois de őp $\gamma\llcorner\alpha$ et de ses composés, quitte à répéter parfois certaines constatations bien connues.

Université de Liège

André MOTTE

Vinciane PIRENNE-DELFORGE aspirant du F.N.R.S.

Place du XX-Août, 32

B - 4000 LIÈGE

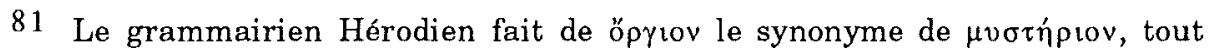
comme certains lexicographes. 